\title{
SOME ABSTRACT CAUCHY PROBLEMS IN EXCEPTIONAL CASES
}

\author{
LOUIS R. BRAGG ${ }^{1}$
}

\begin{abstract}
Let $\mathbf{X}$ be a Banach space and let $A=B^{2}$ in which $B$ is the infinitesimal generator of a strongly continuous group in $X$ with dense domain $\mathscr{D}(A)$. This paper develops solutions of the abstract Euler-PoissonDarboux problem

$$
\begin{aligned}
u_{t t}(t)+\frac{1-2 m}{t} u_{t}(t)=A u(t), & t>0, \quad m=1,2,3, \ldots, \\
\|u(t)-\phi\| \rightarrow 0 \text { as } t \rightarrow 0, & \phi \in \mathscr{D}\left(A^{r}\right), \quad r>m,
\end{aligned}
$$

and associated Cauchy problem in terms of solutions of related abstract wave problems. Connections between solutions of certain abstract hypergeometric equations play an important role in these developments. J. B. Diaz and $\mathrm{H}$. Weinberger and $\mathrm{E}$. K. Blum have obtained solutions of the standard Euler-Poisson-Darboux problem (i.e. $A=\Delta_{n}$, the Laplacian) in the exceptional cases.
\end{abstract}

1. Introduction. Let $\mathbf{X}$ be a Banach space with norm \|\| , let $A=B^{2}$ in which $B$ is the infinitesimal generator of a strongly continuous group in $\mathbf{X}$ with dense domain $\mathscr{D}(A)$ (see [10]), and let $k$ be a real constant. We shall be concerned with constructing solutions to the singular abstract Cauchy problem

$$
\begin{array}{rlrl}
u^{\prime \prime}(t)+(k / t) u^{\prime}(t)-A u(t) & =0, & & u(t) \in \mathbf{X}, \quad t>0, \\
u(0+)=\phi, & & u^{\prime}(0+)=0, \quad \phi \in \mathbb{D}\left(A^{r}\right),
\end{array}
$$

when $k=-1,-3,-5, \ldots$ as well as to closely related problems. In this, we understand that the initial conditions are taken on in the sense of the norm, i.e., $\|u(t)-\phi\| \rightarrow 0$ as $t \rightarrow 0$, and that $r$ is a large positive integer. Following the terminology used in the case of the standard Euler-Poisson-Darboux equation, we refer to these values of $k$ as the exceptional values. J. A. Donaldson [13] established the existence and uniqueness of solutions of (1.1) when $k \geqslant 0$ while exhibiting nonuniqueness when $k<0$. The shifting relations developed in [5] and [7] permit one to obtain solutions of (1.1) for $k<0$

\footnotetext{
Received by the editors October 19, 1976.

AMS (MOS) subject classifications (1970). Primary 47E05; Secondary 47D10.

Key words and phrases. Banach space, strongly continuous group, singular Cauchy problems, Euler-Poisson-Darboux equation, hypergometric equations, logarithmic solutions.

' Supported by the National Science Foundation Grant No. MCS 76-06450.
} 
but not exceptional. In the exceptional cases, a method is given in [5] that permits one to construct a simple logarithmic solution if $A^{(3-k) / 2} \cdot \phi=0$ (and a nonlogarithmic solution if $\left.A^{(1-k) / 2} \cdot \phi=0\right)$. These conditions are generalizations of the Weinstein polyharmonic requirement (see below). Additional results pertaining to (1.1) are given in [14].

The problem (1.1) is, of course, a generalization of the familiar problem for the Euler-Poisson-Darboux equation:

$$
\begin{aligned}
& u_{t t}\left(x_{1}, \ldots, x_{n} ; t\right)+\frac{k}{t} u_{t}\left(x_{1}, \ldots, x_{n} ; t\right) \\
&-\Delta_{n} u\left(x_{1}, \ldots, x_{n} ; t\right)=0, \quad t>0, \\
& u\left(x_{1}, \ldots, x_{n} ; 0+\right)=f\left(x_{1}, \ldots, x_{n}\right), \quad u_{t}\left(x_{1}, \ldots, x_{n} ; 0+\right)=0,
\end{aligned}
$$

$\Delta_{n}=D_{x_{1}}^{2}+\cdots+D_{x_{n}}^{2}$, which has been the subject of an extensive number of research articles. Among the most notable contributions are those by $L$. Ásgeirsson [1] for the case $k=n-1$; A. Weinstein [19] for the case $k>n-1$ and [20] for the case $k<n-1, k$ not exceptional; J. B. Diaz and $\mathrm{H}$. Weinberger [12] for the case $k<n-1$ including exceptional values and E. K. Blum [2], [3] for $k<0$. Weinstein pointed out the significance of requiring $f\left(x_{1}, \ldots, x_{n}\right)$ to be polyharmonic in developing solutions $(1.2)$ in the exceptional cases. Diaz and Weinberger employed an analytic continuation technique to obtain solutions of (1.2) in the exceptional cases for more general choices for $f\left(x_{1}, \ldots, x_{n}\right)$ while Blum obtained solutions in such cases by reducing their development to the treatment of (1.2) with $k=1$. Following either procedure, the solutions obtained involve logarithmic terms. The methods used in these latter two papers are specifically tied in with the Laplacian operator (such as spherical means, etc.) and cannot be generalized to treat (1.1) in the exceptional cases.

Our treatment of (1.1) in these exceptional cases is based upon an elementary relation connecting a solution of an abstract Cauchy problem involving an "exceptional" hypergeometric equation (of the form $t w$ " $(t)$ $\left.-m w^{\prime}(t)-A w(t)=0, m=0,1,2, \ldots\right)$ to the solution of an abstract Cauchy problem involving a nonexceptional hypergeometric equation. This relation is given in $\$ 2$ and involves logarithmic terms. Through the use of Taylor's theorem, this relation is then used to construct solutions of (1.1) in the exceptional cases. The reduction of the problem to a single nonexceptional case is reminiscent of Blum's approach in the case of (1.2). Finally, by employing a theorem on related partial differential equations from [5], this relation is used in $\$ 3$ to solve a somewhat different singular Cauchy problem in an exceptional case.

It should be mentioned that one can use a variety of forms of logarithmic solutions of standard "exceptional" hypergeometric equations to derive solutions of (1.1) for $k=-1,-3,-5, \ldots$ because of nonuniqueness. Such logarithmic solutions are given, for example, in [17]. The form of the result we 
use ties in more closely with our earlier work on related partial differential equations.

2. Solutions of (1.1). The result to be employed for the construction of solutions of (1.1) in the exceptional cases is given in

TheOREM 2.1. Let $\mathbf{X}$ be a Banach space with norm \|\| and let $A=B^{2}$ in which $B$ is the infinitesimal generator of a strongly continuous group in $\mathbf{X}$ with dense domain $\mathscr{D}(A)$. Let $U(\sigma)$ be a solution to the abstract Cauchy problem

$$
\begin{array}{rlrl}
\sigma U^{\prime \prime}(\sigma)+U^{\prime}(\sigma)-A U(\sigma) & =0, & & \sigma>0, \\
U(0+) & =\phi, \quad & \phi \in \mathscr{D}\left(A^{r}\right), r \text { any positive integer }
\end{array}
$$

and define $V(\sigma)$ by the relation

$$
V(\sigma)=U(\sigma)+\{\sigma \ln \sigma\} U^{\prime}(\sigma)-2 \sigma D_{\sigma} \int_{0}^{1} \frac{U(\sigma)-U(\sigma \xi) d \xi}{1-\xi}
$$

Then $V(\sigma)$ is a solution to the abstract Cauchy problem

$$
\begin{aligned}
\sigma V^{\prime \prime}(\sigma)-A V(\sigma) & =0, \quad \sigma>0, \\
V(0+) & =\phi .
\end{aligned}
$$

This formula for $V(\sigma)$ can be derived from the integral (3.1) of [7] by making use of finite and logarithmic parts of divergent integrals (see [9]) but the derivation is rather lengthy because of technical details. For the purposes of brevity, we verify the result directly.

Proof. Rewriting (2.2), we have

$$
V(\sigma)=U(\sigma)+\{\sigma \ln \sigma\} U^{\prime}(\sigma)-2 \int_{0}^{1} \frac{\sigma U^{\prime}(\sigma)-\sigma \xi U^{\prime}(\sigma \xi)}{1-\xi} d \xi .
$$

The integral in (2.2) is taken in the strong Riemann sense.

Differentiating this with respect to $\sigma$, we find that

$$
\begin{aligned}
V^{\prime}(\sigma)= & 2 U^{\prime}(\sigma)+(\ln \sigma) U^{\prime}(\sigma)+(\sigma \ln \sigma) U^{\prime \prime}(\sigma) \\
& -2 \int_{0}^{1} \frac{U^{\prime}(\sigma)+\sigma U^{\prime \prime}(\sigma)-\xi U^{\prime}(\sigma \xi)-\sigma \xi^{2} U^{\prime \prime}(\sigma \xi)}{1-\xi} d \xi \\
= & 2 U^{\prime}(\sigma)+A\{(\ln \sigma) U(\sigma)\}-2 A \int_{0}^{1} \frac{U(\sigma)-\xi U(\sigma \xi)}{1-\xi} d \xi .
\end{aligned}
$$

The last member of this equality follows by eliminating all of the second derivatives of $U$ from the second member of this equality by using the 
equation in (2.1) and by making use of the strong integrability to remove $A$ from under the sign of integration [10]. Similarly,

$$
\begin{aligned}
\sigma V^{\prime \prime}(\sigma)= & 2 \sigma U^{\prime \prime}(\sigma)+A U(\sigma)+A\left\{(\sigma \ln \sigma) U^{\prime}(\sigma)\right\} \\
& -2 \sigma A \int_{0}^{1} \frac{U^{\prime}(\sigma)-\xi^{2} U^{\prime}(\sigma \xi)}{1-\xi} d \xi \\
A V(\sigma)= & A U(\sigma)+A\{(\sigma \ln \sigma)\} U^{\prime}(\sigma)-2 A \int_{0}^{1} \frac{\sigma U^{\prime}(\sigma)-\sigma \xi U^{\prime}(\sigma \xi)}{1-\xi} d \xi
\end{aligned}
$$

A comparison of the right members of these two relations shows that the equation in (2.3) is satisfied provided that

$$
\sigma U^{\prime \prime}(\sigma)-A \int_{0}^{1} \xi U_{\xi}(\sigma \xi) d \xi=0 .
$$

Upon replacing $U^{\prime \prime}(\sigma)$ by $A U(\sigma)-U^{\prime}(\sigma)$ and evaluating the integral in (2.4) by parts, $(2.4)$ becomes

$$
\begin{aligned}
-U_{\sigma}(\sigma)+A \int_{0}^{1} U(\sigma \xi) d \xi & =-U_{\sigma}(\sigma)+\int_{0}^{1} A U(\sigma \xi) d \xi \\
& =-U_{\sigma}(\sigma)+\int_{0}^{1}\left[\sigma \xi U^{\prime \prime}(\sigma \xi)+U^{\prime}(\sigma \xi)\right] d \xi \\
& =-U_{\sigma}(\sigma)+\int_{0}^{1} \frac{\partial}{\partial \sigma}\left[\sigma U^{\prime}(\sigma \xi)\right] d \xi \\
& =-U_{\sigma}(\sigma)+\frac{d}{d \sigma} \int_{0}^{1} U_{\xi}(\sigma \xi) d \xi=0 .
\end{aligned}
$$

Hence, equation (2.4) holds so that the equation in (2.3) is satisfied. It is easy to verify that the initial condition in (2.3) is satisfied by use of a simple norm inequality.

Corollary 2.1. Given the conditions of Theorem 2.1, let $U(\sigma)$ denote a solution of

$$
\begin{aligned}
\sigma U^{\prime \prime}(\sigma)+U^{\prime}(\sigma)-A U(\sigma) & =0, \quad \sigma>0, \\
U(0+) & =(-1)^{m} A^{m} \phi / m !, \quad \phi \in \mathscr{D}\left(A^{r}\right),
\end{aligned}
$$

in which $r$ is any positive integer $>m$ and let

$$
V(\sigma)=U(\sigma)+(\sigma \ln \sigma) U^{\prime}(\sigma)-2 \sigma D_{\sigma} \int_{0}^{1} \frac{U(\sigma)-U(\sigma \xi)}{1-\xi} d \xi
$$

Then the function 


$$
\begin{aligned}
W(\sigma)= & \phi-\frac{(\sigma A) \phi}{1 ! m}+\frac{(\sigma A)^{2} \phi}{2 ! m(m-1)}+\cdots \\
& +\frac{(-1)^{m-1}(\sigma A)^{m-1} \phi}{(m-1) ! m !}+\int_{0}^{\sigma} \frac{V(\xi)(\sigma-\xi)^{m-1}}{(m-1) !} d \xi
\end{aligned}
$$

is a solution of the abstract Cauchy problem

$$
\begin{aligned}
\sigma W^{\prime \prime}(\sigma)-m W^{\prime}(\sigma) & =A W(\sigma), \quad \sigma>0, \\
W(0+) & =\phi .
\end{aligned}
$$

Proof. This follows from Theorem 2.1 by an application of Taylor's theorem. The reason for not specifying the value of $r$ earlier is that its choice is clearly dependent upon the equation under consideration.

With the change of variables $\sigma=t^{2} / 4$ in (1.1), problem (1.1) becomes

$$
\begin{aligned}
\sigma \tilde{u}_{\sigma \sigma}(\sigma)+((k+1) / 2) \tilde{u}_{\sigma}(\sigma)-A \tilde{u}(\sigma) & =0, \\
\tilde{u}(0+) & =\phi, \quad \phi \in \mathscr{Q}\left(A^{r}\right),
\end{aligned}
$$

in which $\tilde{u}(\sigma)$ is a solution function corresponding to $u(t)$ under the stated change of variables. Then, if $k=-(2 m+1)$, the equation in (2.9) is precisely the equation involved in problem (2.8). Hence, $\tilde{u}(\sigma)$ is given by (2.7) and a solution of $(1.1)$ for $k=-(2 m+1)$ is $u(t)=\tilde{u}\left(t^{2} / 4\right)$.

Taking $\sigma=t^{2} / 4$, it follows that the function $U$ occurring in Corollary 2.1 is a solution of the abstract Euler-Poisson-Darboux problem

$$
\begin{gathered}
U_{t t}\left(t^{2} / 4\right)+(1 / t) U_{t}\left(t^{2} / 4\right)-A U\left(t^{2} / 4\right)=0, \\
U(0+)=(-1)^{m} A^{m} \phi / m !,\left.\quad U_{t}\left(t^{2} / 4\right)\right|_{t=0+}=0 .
\end{gathered}
$$

This function $U\left(t^{2} / 4\right)$ can be expressed in terms of the solution $w(t)$ of the abstract wave problem

$$
\begin{aligned}
w^{\prime \prime}(t)-A w(t) & =0, \quad t>0, \\
w(0+) & =(-1)^{m} A^{m} \phi / m !, \quad w^{\prime}(0+)=0,
\end{aligned}
$$

by means of the integral $[4$, p. 609$]$

$$
U\left(t^{2} / 4\right)=\frac{2}{\pi} \int_{0}^{t} \frac{w(\eta)}{\sqrt{t^{2}-\eta^{2}}} d \eta
$$

A solution of (2.11) exists if $A^{m} \phi \in \mathscr{D}(A)$. There exist explicit constructions for the function $w(t)$ in (2.11) (see [15], [16], [6], [11]) in terms of the group generated by $B$. 
3. A further application. Consider the singular Cauchy problem

$$
\begin{aligned}
Z_{t t}(x, t) & =Z_{x x}(x, t)+(k / t) Z_{x}(x, t), \\
Z(x, 0) & =\phi(x), \quad Z_{t}(x, 0)=0,
\end{aligned}
$$

in which $\phi(x)$ is taken to be a bounded analytic function. The equation in this resembles the Euler-Poisson-Darboux equation in one space variable except that the term $t^{-1}$ multiplies a space derivative term rather than a time derivative term. For $k>0$, we reduce the solution of (3.1) to the solution of a problem analogous to $(2.1)$.

Introduce the change of variables $t=\xi / 2$ in (3.1) followed by the changes of variables $y=x-\xi / 2, z=\xi$. Then (3.1) becomes

$$
\begin{aligned}
z D_{z}\left(z D_{z}-1\right) \tilde{Z}(y, z)-z D_{y}\left(z D_{z}+k / 2\right) \tilde{Z}(y, z) & =0, \\
\tilde{Z}(y, 0) & =\phi(y)
\end{aligned}
$$

in which $Z(y, z)$ corresponds to $Z(x, t)$ under the stated changes of independent variables. Using Theorem 3.1 of [5], it follows that, for $k>0$,

$$
\mathcal{Z}(y, z)=\frac{1}{\Gamma(k / 2)} \int_{0}^{\infty} e^{-\sigma} \sigma^{k / 2-1} V(y, z \sigma) d \sigma
$$

where $V(y, z)$ is a solution of the problem

$$
z V_{z z}(y, z)-D_{y} V(y, z)=0, \quad V(y, 0+)=\phi(y)
$$

obtained by deleting the operator factor $\left(z D_{z}+k / 2\right)$ from the equation in problem (3.2). But the equation in (3.4) is precisely the equation in (2.3) with $A$ taken to be the derivative operator $D_{y}$. Therefore, a solution of (3.4) can be obtained by selecting $U(y, z)$ to be a solution of

$$
\begin{aligned}
z U_{z z}(y, z)+U_{z}(y, z)-D_{y} U(y, z) & =0, \\
U(y, 0+) & =\phi(y)
\end{aligned}
$$

and forming the function $V(y, z)$ as in (2.2).

The function $U(y, z)$ in (3.5) can be obtained from the solution of the "wave" problem

$$
\begin{aligned}
W_{z z}(y, z)-D_{y} W(y, z) & =0, \\
W(y, 0+) & =\phi(y), \quad W_{z}(y, 0+)=0,
\end{aligned}
$$

by means of (2.12). But the function $W(y, z)$ is related to the solution function of the problem

$$
\tilde{W}(y, z)-D_{y} \tilde{W}(y, z)=0, \quad \tilde{W}(y, 0+)=\phi(y)
$$

by means of the relation 


$$
W(y, z)=\Gamma\left(\frac{1}{2}\right) z \mathcal{L}_{s}^{-1}\left\{s^{-1 / 2} \tilde{W}(y, 1 / 4 s)\right\}_{s \rightarrow z^{2}}
$$

in which $\mathfrak{E}_{s}^{-1}\{\}_{s \rightarrow z^{2}}$ denotes the inverse Laplace transform with $s$ the variable of the transform and $z^{2}$ the variable of inversion (see (2.2) of [8]). However, it readily follows that $\tilde{W}(y, z)=\phi(y+z)$ so that all of the functions needed to obtain a solution of (3.1) have been defined. The function $Z(x, t)$ can now be readily obtained from them.

Suppose one were to replace the partial differential equation in (3.1) by the equation

$$
Z_{t t}(x, t)=t^{-2 m /(m+1)} Z_{x x}(x, t)+\nu t^{-m /(m+1)-1} Z_{x}(x, t),
$$

$m$ a nonnegative integer. Upon introducing the change of variables $\xi$ $=2(m+1) t^{1 /(m+1)}$ followed by the changes $y=x-\xi / 2, z=\xi$, this equation becomes

$$
z D_{z}\left(z D_{z}-m-1\right) \tilde{Z}(y, z)-z D_{y}\left(z D_{z}+\frac{\nu(m+1)-m}{2}\right) \tilde{Z}(y, z)=0
$$

and would replace the equation in (3.2). Using the above procedures, it follows, for $\nu>m /(m+1)$, that

$$
\mathcal{Z}(y, z)=\left[\Gamma\left(\frac{\nu(m+1)-m}{2}\right)\right]^{-1} \int_{0}^{\infty} e^{-\sigma} \sigma^{(\nu(m+1)-m) / 2-1} V(y, z \sigma) d \sigma
$$

in which $V(y, z)$ is a solution of the problem

$$
z V_{z z}(y, z)-m V_{z}(y, z)-D_{y} V(y, z)=0, \quad V(y, 0+)=\phi(y) .
$$

But this problem can be solved by an application of Corollary 2.1.

\section{BIBLIOGRAPHY}

1. L. Ásgeirsson, Uber eine Mittelwerteigenschaft von Lösungen homogener linearer partiellen Differential gleichungen 2 ordnung mit konstanten Koeffizienten, Math. Ann. 113 (1937), 321-346.

2. E. K. Blum, The solutions of the Euler-Poisson-Darboux equation for negative values of the parameter, Duke Math. J. 21 (1954), 256-269.

3. - The Euler-Poisson-Darboux equation in the exceptional cases, Proc. Amer. Math. Soc. 5 (1954), 511-520.

4. L. R. Bragg, Fundamental solutions and properties of solutions of the initial value radial EulerPoisson-Darboux problem, J. Math. Mech. 18 (1969), 607-616.

5. Hypergometric operator series and related partial differential equations, Trans. Amer. Math. Soc. 143 (1969), 319-336.

6. - Singular, non-homogeneous abstract Cauchy and Dirichlet type problems related by a generalized Stieltjes transform, Indiana Univ. Math. J. 24 (1974), 183-195.

7. - The Riemann-Liouville integral and parameter shifting in a class of linear abstract Cauchy problems, SIAM J. Math. Anal. 7 (1976), 1-12.

8. L. R. Bragg and J. W. Dettman, An operator calculus for related parital differential equations, J. Math. Anal. Appl. 22 (1968), 261-271.

9. F. J. Bureau, Divergent integrals and partial differential equations, Comm. Pure Appl. Math. 12 (1954), 143-202.

10. P. Butzer and H. Berens, Semi-groups of operators and approximations, Springer-Verlag, New York, 1967. 
11. J. W. Dettman, Saturation theorems connected with the abstract wave equation, SIAM J. Math. Anal. (to appear).

12. J. B. Diaz and H. F. Weinberger, A solution of the singular initial value problem for the Euler-Poisson-Darboux equation, Proc. Amer. Math. Soc. 4 (1953), 703-715.

13. J. A. Donaldson, A singular abstract Cauchy problem, Proc. Nat. Acad. Sci. U.S.A. 66 (1970), 269-274.

14. - An operator calculus for a class of abstract operator equations, J. Math. Anal. Appl. 37 (1972), 167-184.

15. H. O. Fattorini, Ordinary differential equations in linear topological spaces. I, J. Differential Equations 5 (1968), 72-105.

16. R. Hersh, Explicit solutions of a class of higher order abstract Cauchy problems, J. Differential Equations 8 (1970), 570-579.

17. Y. Luke, The special functions and their approximations, Vol. 1, Academic Press, New York, 1969.

18. S. G. Mikhlin (Editor), Linear equations of mathematical physics, Holt, Rinehart, and Winston, New York, 1967.

19. A. Weinstein, Sur le problème de Cauchy pour l'equation de Poisson et l'equation des ondes, C. R. Acad. Sci. Paris 234 (1952), 2584-2585.

20. - On the wave equation and the equation of Euler-Poisson, Proc. Sympos. Appl. Math., vol. 5, Amer. Math. Soc., Providence, R.I., 1954, pp. 137-147.

Departmant of Mathematics, OAKLANd University, Rochester, Michigan 48063 